\title{
TRADUÇÃO E VALIDAÇÃO PARA A LÍNGUA PORTUGUESA DE QUESTIONÁRIO UTILIZADO EM ESTUDOS DE CONSUMIDOR ${ }^{1}$
}

\author{
Rosires DELIZA ${ }^{2, *}$, Amauri ROSENTHAL ${ }^{2}$, Maria Cristina da COSTA ${ }^{3}$
}

\begin{abstract}
RESUMO
O presente trabalho objetivou disponibilizar para a língua portuguesa o questionário originalmente desenvolvido em inglês e denominado Need for Cognition (NFC). Tal questionário tem considerável importância no desenvolvimento de estudos do consumidor, uma vez que possibilita segmentar a população de acordo com determinada característica, ou seja, o quanto gostam de se engajar no pensar. O questionário foi traduzido utilizando a metodologia denominada back translation e validado objetivando disponibilizar para a lingua portuguesa tal ferramenta, a qual auxilia na interpretação e no entendimento da atitude e comportamento do consumidor frente a um determinado produto ou questão. A maioria das escalas e questionários disponiveis foram desenvolvidos em outros países e, como é reconhecido, um processo de adaptação cultural e posterior validação da nova versão são requeridos para serem usados em culturas diferentes daquela para qual foi originalmente desenvolvida. Assim, faz-se necessário desenvolver o instrumento no próprio idioma, ou traduzi-lo e validá-lo. A técnica back translation garante tradução precisa e confiável do instrumento. A versão original em inglês foi traduzida para o português independentemente por três indivíduos bilingües. O instrumento traduzido foi revisado aplicando as duas versões a um grupo de 15 individuos bilingües, a fim de checar a equivalência da tradução.

Palavras-chave: comportamento; consumidor; tradução; validação; lingua portuguesa; atitude.
\end{abstract}

\section{SUMMARY}

TRANSLATION AND VALIDATION INTO PORTUGUESE LANGUAGE OF SCALE USED IN CONSUMER STUDIES. This work aimed at making available to the Portuguese language the questionnaire developed in English, and named Need for Cognition (NFC). This questionnaire has high importance on the development of consumer studies because it allows the segmentation of the population according to a specific characteristic, i.e. how much people engage in and enjoy thinking. The questionnaire has been translated using the methodology back translation, and validated in order to make available a tool which helps the consumer interpretation and understanding towards a product or matter. As the majority of the scales and questionnaires have been developed in countries different from those where they should be applied, a cultural adaptation and validation of the new version are required before using them. Thus, either it is necessary to develop an instrument in the specific language, or translate and validate it. Back translations ensures a reliable and precise instrument translation. The English version was translated and back translated independently by three bilinguals. The Portuguese version was checked for translation equivalency through presentation of both versions to a group of 15 bilinguals individuals. Keywords: consumer behaviour; translation; validation; Portuguese language.

\section{1 - INTRODUÇÃO}

A relação entre variáveis de personalidade com o comportamento do indivíduo é do interesse de várias áreas relacionadas ao entendimento do processo pelo qual estas variáveis influenciam a preferência e a escolha de um produto pelo consumidor. A possibilidade de segmentar uma população de acordo com as características individuais das pessoas, seja através das diferenças de personalidade, ou de atitudes em relação a aspectos específicos, aparece como uma possibilidade promissora para o desenvolvimento de campanhas promocionais, embalagens e rótulos, os quais podem contribuir para uma maior satisfação do consumidor com o produto.

Tal segmentação pode ser atingida utilizando-se ferramentas apropriadas que auxiliam na caracterização do indivíduo. Muitos fatores podem ser responsáveis pelas diferenças de percepção entre consumidores, mesmo quando estes são submetidos a estímulos idênticos. Segundo o Modelo da Probabilidade da Elaboração -

1. Recebido para publicação em 20/02/2001. Aceito para publicação em 15/05/2002.

2. EMBRAPA Agroindústria de Alimentos. Av. das Américas, 29501. CEP 23020-470 Rio de Janeiro - RJ. E-mail: rodeliza@ctaa.embrapa.br

3. Departamento de Tecnologia de Alimentos da UFRRJ, Rio de Janeiro$R J, B R$.

* A quem a correspondência deve ser enviada.
Elaboration Likelihood Model (ELM) - existem duas rotas de persuasão: a central, através da avaliação racional e crítica dos argumentos e a periférica, a qual é baseada em simples sugestão afetiva, sem incluir o processamento cognitivo. Utilizando a rota central, o consumidor usa informação relevante para avaliar o produto e, seguindo a rota periférica, usa apenas simples sugestões ou influência do contexto, sem considerar os atributos reais do produto [3]. Muitas variáveis podem moderar a escolha da rota cognitiva, e entre elas estão o NFC (Need for Cognition), humor, cansaço, envolvimento com a compra.

Need for Cognition (NFC) representa a tendência do indivíduo em se engajar e gostar de atividade que exige esforço analítico [5] e tem sido utilizado em pesquisas de mercado para examinar o efeito de mensagens persuasivas através de propaganda, rótulo ou informação no comportamento do consumidor [2]. Tal ferramenta foi desenvolvida por CACIOPPO, PETTY, KAO [4] na forma de questionário contendo 18 itens, o qual é apresentado ao consumidor como frases afirmativas, onde o indivíduo deve expressar o quanto concorda ou discorda de cada uma delas. A escala utilizada é de nove pontos variando de 1 (concordância muito intensa) a 9 (discordância muito intensa). A soma das notas para os 18 itens vai classificar o indivíduo como baixo, médio ou alto na variável NFC. Segundo HAUGTVEDT, PETTY, CACIOPPO [8], indivíduos com alto NFC utilizam a rota central e tendem, portanto, a avaliar os argumentos contidos na propa- 
ganda e rótulos mais extensivamente, enquanto os individuos com baixo NFC, os quais devem seguir a rota periférica, valorizam aspectos não relacionados ao produto. DELIZA [6] demonstrou que a informação contida no rótulo foi o aspecto mais importante na avaliação do produto pelos consumidores com alto NFC, enquanto que a cor e ilustração alcançou maior importância relativa para os consumidores com baixo NFC.

Geralmente, os instrumentos utilizados para avaliar a atitude do consumidor frente a diversos aspectos relacionados à saúde, educação, alimentos, etc. foram desenvolvidos em países cuja língua mãe não é o português. A maioria deles foi desenvolvida nos Estados Unidos e Inglaterra e, como é reconhecido, um processo de adaptação cultural e posterior avaliação da validade da nova versão são requeridos antes que o instrumento possa ser recomendado para ser usado em culturas diferentes daquela para a qual foi originalmente desenvolvida. Assim, a falta de um instrumento de pesquisa na lingua desejada, acena para duas possibilidades: desenvolvimento de instrumentos no próprio idioma, ou utilização daqueles já existentes, após traduzi-los e validálos. Uma tradução adequada deve considerar todo o sentido contido na versão original, preservando e transpondo o impacto, significado, idéias, forma e total valor do instrumento original para o novo contexto lingüístico; alcançando, portanto, perfeição no estilo e na lingüística [9]. Versões de vários instrumentos desenvolvidos em inglês foram obtidas em espanhol, coreano e hebraico utilizando metodologia similar $[1,7,11]$.

O presente trabalho objetivou disponibilizar para a lingua portuguesa, o questionário originalmente desenvolvido em inglês Need for Cognition (NFC) [5], seguindo o processo de adaptação que inclui tradução, back translation, revisão e aplicação do questionário para uma amostra bilíngüe da população. Esse processo permite a avaliação da equivalência entre as versões inglesa e portuguesa.

\section{2 - MATERIAL E MÉTODOS}

\section{1 - Método}

As 18 questões que compõem a variável Need for Cognition [4] foram traduzidas para o português por três tradutores bilingües trabalhando independentemente, gerando, portanto, três versões em português. Tais versões foram traduzidas para o inglês por outros indivíduos bilingües, conforme sugere a metodologia back translation [9]. Em seguida, foram comparadas com o original em inglês e discutidas a fim de se procederem os ajustes necessários para se obter uma única versão apropriada do instrumento em português, garantindo, assim, a equivalência de sentido (Figura 1). Esta técnica garante tradução mais precisa e confiável do instrumento.

Os 18 itens que compõem o questionário Need for Cognition foram respondidos utilizando escala de nove pontos, a qual variou de 1 (concordância muito intensa) a 9 (discordância muito intensa), passando pelo ponto 5 (nem concordo e nem discordo), seguindo a versão original [4].

\section{2 - Individuos}

Uma maneira de comparar as propriedades psicométricas do instrumento na linguagem original e na nova linguagem é administrar ambas as versões para indivíduos fluentes em ambos idiomas [11]. Assim, dezesseis indivíduos bilingües que lêem e entendem os dois idiomas foram recrutados para participar do estudo. Dentre os participantes, quatorze deles residiram por pelo menos 3 anos em país de lingua inglesa e duas mulheres, apesar de nunca terem morado em tais países, possuem reconhecida fluência no idioma. Metade dos participantes pertenciam ao sexo masculino e metade ao feminino, com idade variando entre 29 e 54 anos. Os indivíduos foram aleatoriamente divididos em grupos 1 e 2, sendo que o grupo 1 recebeu primeiro a versão em português e após um intervalo de 5 dias respondeu a versão em inglês, seguindo o recomendado por PRIETO [9]. O grupo 2 trabalhou na versão inglesa e, posteriormente, na portuguesa, obedecendo o mesmo intervalo de tempo entre as respostas.

\section{3 - Análise estatística}

A equivalência entre a versão portuguesa e inglesa da variável NFC foi assim verificada:

\subsection{1 - Equivalência de conceito}

As respostas dos participantes para cada um dos 18 itens que compõem a versão inglesa da escala Need for Cognition foram comparadas com as respectivas respostas dadas à versão portuguesa da mesma ferramenta utilizando teste $t$ e o coeficiente de correlação de Pearson, avaliando-se, assim, a equivalência conceitual da tradução. Um coeficiente de correlação mínimo de 0,50 foi considerado aceitável [11].

\subsection{2 - Equivalência de escala}

Avaliou o quão semelhante foram as notas dadas pelos mesmos indivíduos nas versões original (inglês) e traduzida (português). Tal equivalência foi calculada segundo OQUENDO et al. [10] para cada indivíduo em termos da proporção de concordância entre as duas versões para as 18 questões do questionário e computada como índice de concordância. Alto índice de concordância indica alta equivalência entre a adaptação em português e a versão inglesa do questionário NFC.

\section{3 - RESULTADOS E DISCUSSÃO}

A Figura 1 apresenta a versão em português do questionário NFC.

A Tabela 1 apresenta os resultados do teste $t$ para cada par de itens (versão em português e em inglês) do instrumento. Pôde-se observar diferença significativa entre as médias para a questão $10(\mathrm{Q} 10$ - The idea of relying on thought to make my way to the top appeals to me) da versão inglesa e a respectiva tradução para o português.

Não houve diferença entre a soma total dos itens das duas versões $(t=-1,50 ; p>0,05)$. 
Nome:

Data:

Por favor indique o quanto você concorda ou discorda de cada uma das seguintes afirmações:

1 Prefiro problemas complexos aos simples.

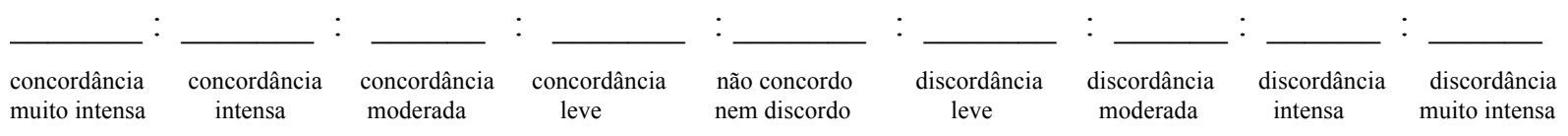

2 Gosto de ter a responsabilidade de lidar com situação que requer muito pensar.

\begin{tabular}{|c|c|c|c|c|c|c|c|c|}
\hline & & & & & & : & & \\
\hline $\begin{array}{l}\text { concordância } \\
\text { muito intensa }\end{array}$ & $\begin{array}{c}\text { concordância } \\
\text { intensa }\end{array}$ & $\begin{array}{l}\text { concordância } \\
\text { moderada }\end{array}$ & $\begin{array}{l}\text { concordância } \\
\text { leve }\end{array}$ & $\begin{array}{l}\text { não concordo } \\
\text { nem discordo }\end{array}$ & $\begin{array}{l}\text { discordância } \\
\text { leve }\end{array}$ & $\begin{array}{l}\text { discordância } \\
\text { moderada }\end{array}$ & $\begin{array}{l}\text { discordância } \\
\text { intensa }\end{array}$ & $\begin{array}{l}\text { discordância } \\
\text { muito intensa }\end{array}$ \\
\hline
\end{tabular}

3 Pensar não é meu passatempo preferido*.

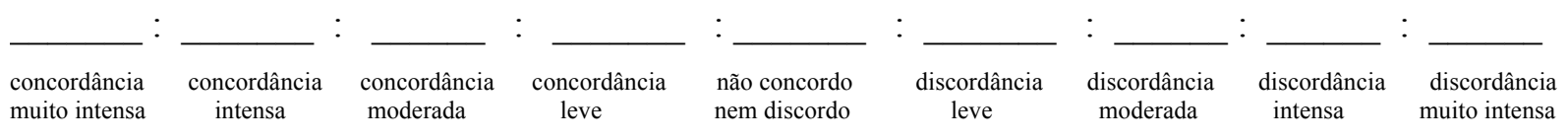

4 Antes faria alguma coisa que requer pouco pensar que alguma coisa que certamente desafiaria minhas habilidades em relação ao pensar*.

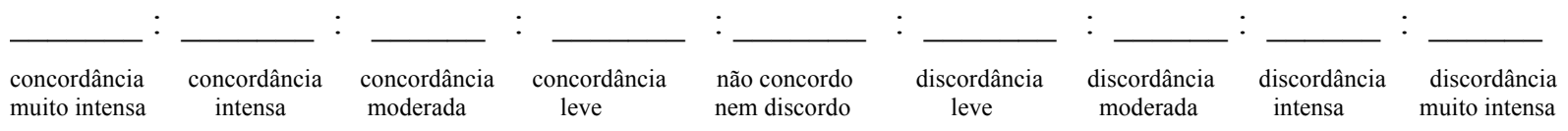

5 Tento antecipar e evitar situações onde exista a provável chance de ter que pensar profundamente sobre alguma coisa*.

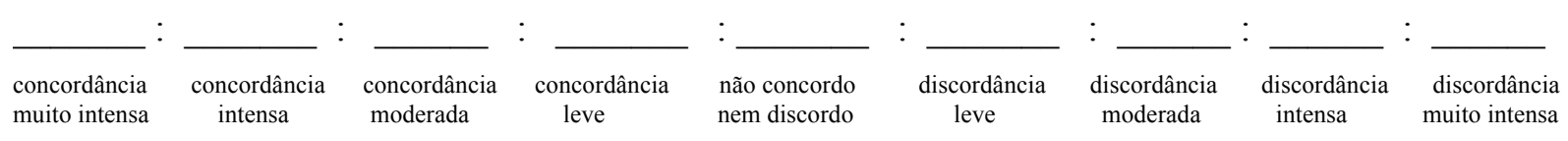

6 Sinto satisfação em ter que ponderar arduamente por muito tempo.

\begin{tabular}{|c|c|c|c|c|c|c|c|c|}
\hline & & & & - & & • & & \\
\hline $\begin{array}{l}\text { concordância } \\
\text { muito intensa }\end{array}$ & $\begin{array}{l}\text { concordância } \\
\text { intensa }\end{array}$ & $\begin{array}{l}\text { concordância } \\
\text { moderada }\end{array}$ & $\begin{array}{l}\text { concordância } \\
\text { leve }\end{array}$ & $\begin{array}{l}\text { não concordo } \\
\text { nem discordo }\end{array}$ & $\begin{array}{l}\text { discordância } \\
\text { leve }\end{array}$ & $\begin{array}{l}\text { discordância } \\
\text { moderada }\end{array}$ & $\begin{array}{l}\text { discordância } \\
\text { intensa }\end{array}$ & $\begin{array}{r}\text { discordância } \\
\text { muito intensa }\end{array}$ \\
\hline
\end{tabular}

7 Apenas me envolvo intensamente quando tenho que me envolver*.

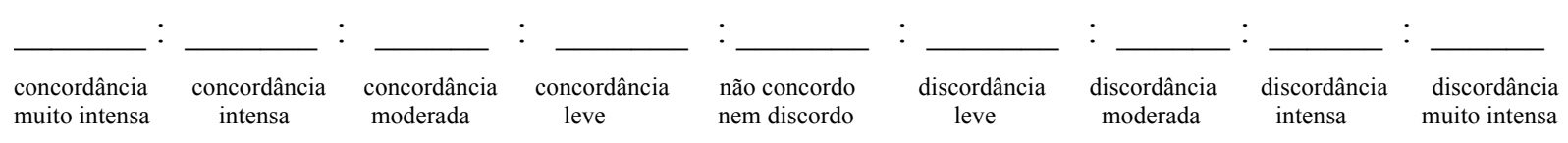

8 Prefiro pensar nos problemas pequenos do dia-a-dia que nos problemas a longo prazo*.

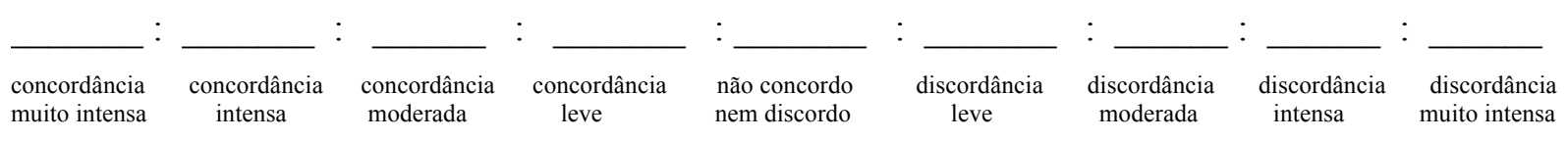

9 Gosto de tarefas que requerem pouco pensar uma vez que as tenha aprendido*.

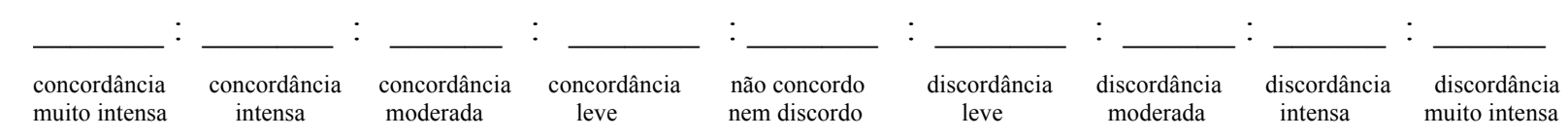

* questões que necessitam reverter as notas.

FIGURA 1. Versão portuguesa do questionário Need for Cognition. 
10 A ideia de utilizar pensamentos para me animar me parece interessante.

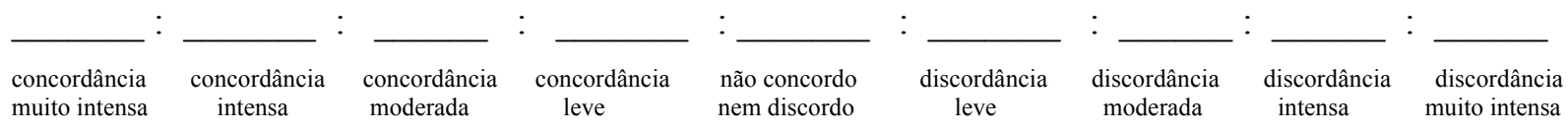

11 Eu realmente gosto de uma tarefa que envolva pensar em novas soluções para os problemas.

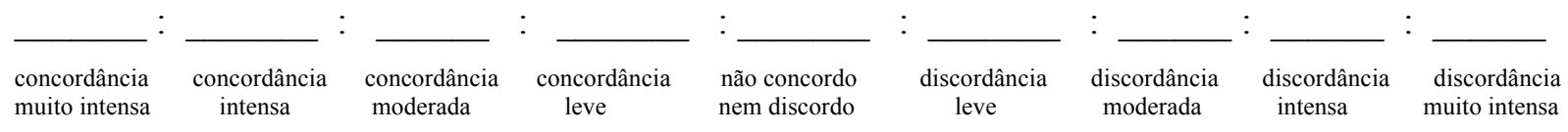

12 Aprender novas maneiras de pensar não me empolga muito*.

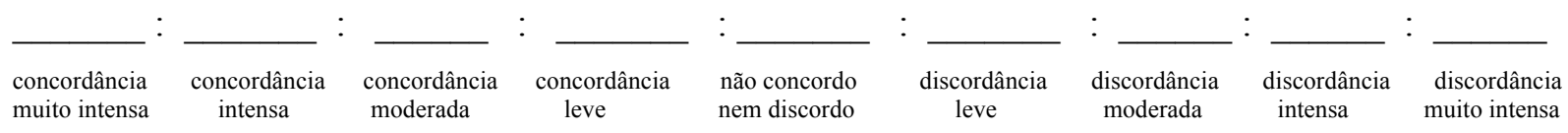

13 Prefiro minha vida repleta de enigmas para resolver.

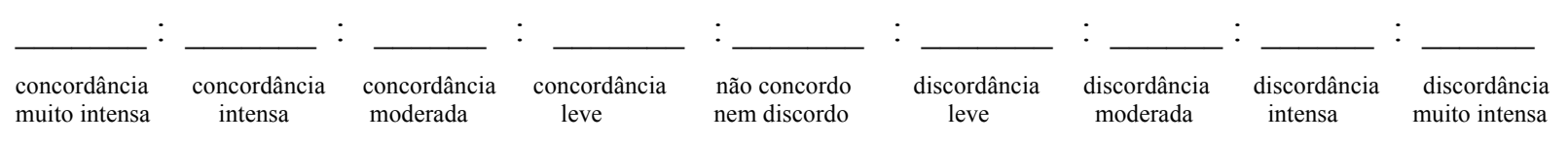

14 Pensar abstratamente me atrai.

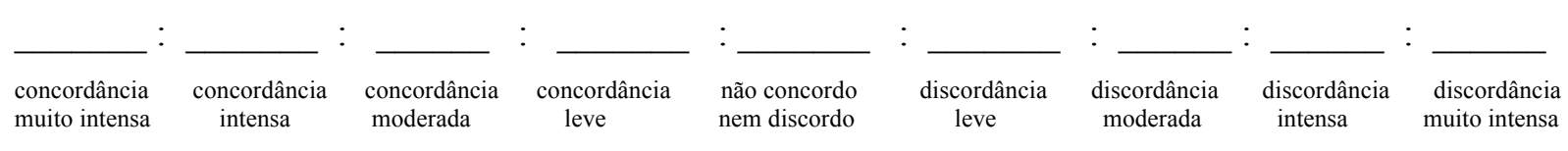

15 Prefiro uma tarefa intelectual, difícil e importante a uma outra que seja importante, mas que não me obrigue a refletir muito.

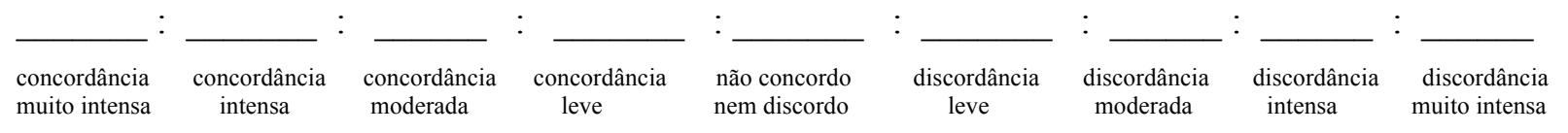

16 Sinto alívio ao invés de satisfação depois de completar uma tarefa que requereu grande esforço mental*.

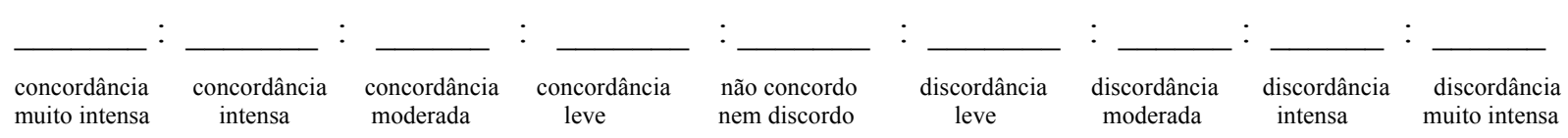

17 É suficiente para mim que o trabalho tenha sido feito, não me importa como e porquê foi feito*.

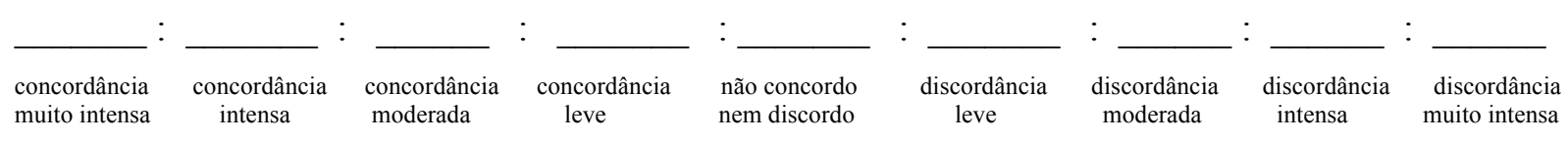

18 Usualmente costumo opinar sobre questões mesmo quando estas não me afetam pessoalmente.

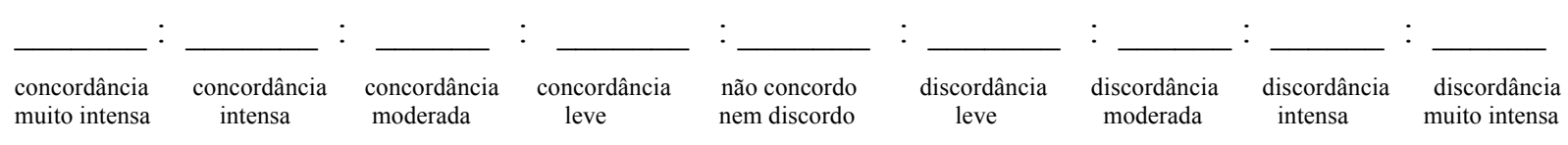

* questões que necessitam reverter as notas.

FIGURA 1. Versão portuguesa do questionário Need for Cognition. 
A Tabela 2 apresenta os resultados do coeficiente de correlação de Pearson, demonstrando que todos os itens tiveram correlação significativa $(p<0,05)$ entre a versão original inglesa e o instrumento traduzido para a lingua portuguesa.

TABELA 1. Teste $t$ para os itens pareados - inglês e português $(\mathrm{N}=16)$.

\begin{tabular}{|c|c|c|c|}
\hline \multirow[b]{2}{*}{ Variável } & \multicolumn{2}{|c|}{ Média } & \multirow[b]{2}{*}{ Valor de $\mathrm{t}$} \\
\hline & Inglês & Português & \\
\hline Par 1 (Q 1) & 4,12 & 3,94 & $-0,25$ \\
\hline Par 2 (Q 2) & 3,25 & 3,00 & $-0,40$ \\
\hline Par 3 (Q 3) & 7,19 & 7,50 & 0,61 \\
\hline Par 4 (Q 4) & 6,93 & 7,12 & 0,34 \\
\hline Par 5 (Q 5) & 6,87 & 7,06 & 0,21 \\
\hline Par 6 (Q 6) & 4,19 & 4,43 & 0,20 \\
\hline Par 7 (Q 7) & 5,69 & 5,93 & 0,21 \\
\hline Par 8 (Q 8) & 5,69 & 6,43 & 1,18 \\
\hline Par 9 (Q 9) & 5,56 & 6,81 & 1,95 \\
\hline Par 10 (Q 10) & 3,30 & 2,23 & $-2,34^{*}$ \\
\hline Par 11 (Q 11) & 2,00 & 1,93 & $-0,21$ \\
\hline Par 12 (Q 12) & 8,12 & 8,19 & 0,31 \\
\hline Par 13 (Q 13) & 4,62 & 4,87 & 0,33 \\
\hline Par 14 (Q 14) & 3,12 & 3,25 & 0,24 \\
\hline Par 15 (Q 15) & 3,75 & 4,00 & 0,39 \\
\hline Par 16 (Q 16) & 5,69 & 7,00 & 1,77 \\
\hline Par 17 (Q 17) & 7,50 & 6,81 & 0,07 \\
\hline Par 18 (Q 18) & 3,93 & 3,62 & $-0,48$ \\
\hline $\begin{array}{l}\text { Par } 19 \text { (soma das } 18 \\
\text { questões) }\end{array}$ & 63,37 & 58,44 & $-1,50$ \\
\hline
\end{tabular}

Em estudo realizado por SON et al. [11], buscando a versão coreana de um instrumento utilizado em pesquisas na área médica, apenas duas questões dentre 15 apresentaram correlação não significativa entre as duas linguagens. Entretanto, este fato não prejudicou a validade e confiabilidade do referido instrumento em coreano. OQUENDO et al. [10], trabalhando na validação para o espanhol de questionário usado em psicologia, demonstraram que 5 dos 48 itens que compunham a ferramenta não apresentaram correlação significativa. Porém, a equivalência foi considerada satisfatória em função da proporção adequada de concordâncias.

Os resultados para a equivalência de escala são mostrados na Tabela 3.

A proporção de concordância entre as versões inglesa e portuguesa foi superior a 0,80 exceto para um item do questionário (Q17), na qual a proporção atingiu 0,75. Os resultados deste estudo foram melhores que aqueles reportados na literatura [10], os quais alcançaram proporção de concordância de 0,60.

Este estudo verificou equivalência na nota total obti- da por indivíduos bilingües na versão original em inglês e na adaptação para o português do questionário NFC. Assim, a versão portuguesa do instrumento pode ser usada em pesquisas de consumidor tendo-se a confiança que as notas obtidas estariam em concordância com aquelas advindas da escala original em inglês.

TABELA 2. Correlação entre os 18 itens na versão inglesa e portuguesa $(\mathrm{N}=16)$.

\begin{tabular}{|c|c|}
\hline Variável & Pearson \\
\hline Par 1 (Q 1) & $0,92^{*}$ \\
\hline Par 2 (Q 2) & $0,78^{*}$ \\
\hline Par 3 (Q 3) & $0,69^{*}$ \\
\hline Par 4 (Q 4) & $0,54^{*}$ \\
\hline Par 5 (Q 5) & $0,67^{*}$ \\
\hline Par 6 (Q 6) & $0,59^{*}$ \\
\hline Par 7 (Q 7) & $0,85^{*}$ \\
\hline Par 8 (Q 8) & $0,81^{*}$ \\
\hline Par 9 (Q 9) & $0,62^{*}$ \\
\hline Par $10(Q 10)$ & $0,74^{*}$ \\
\hline Par 11 (Q 11) & $0,81^{*}$ \\
\hline Par 12 (Q 12) & $0,26^{*}$ \\
\hline Par 13 (Q 13) & $0,81^{*}$ \\
\hline Par 14 (Q 14) & $0,78^{*}$ \\
\hline Par 15 (Q 15) & $0,74^{*}$ \\
\hline Par 16 (Q 16) & $0,86^{*}$ \\
\hline Par 17 (Q 17) & $0,88^{*}$ \\
\hline Par 18 (Q 18) & $0,68^{*}$ \\
\hline
\end{tabular}
$\mathrm{Q}=$ questão no instrumento Need for Cognition.
${ }^{*} \mathrm{p}<0,05$.

As análises estatísticas indicaram que a versão traduzida para o português do questionário original inglês NFC foi semanticamente equivalente. Apenas uma das 18 questões que compõem o instrumento apresentou diferença significativa entre as duas versões, indicando, assim, correlação entre as demais 17 questões em termos de significado.

A disponibilidade do presente instrumento em lingua portuguesa é bastante útil em estudos de consumidor uma vez que possibilita examinar os efeitos de mensagens persuasivas advindas do rótulo, embalagem, propaganda e informação em geral na atitude do indivíduo frente a determinado produto alimentício, em relação a diversas variáveis dependentes (p.ex.: o quanto gostou/ não gostou do produto, intenção de compra, percepção de características sensoriais etc.). Como potencial beneficio prático estaria o entendimento da importância das variáveis relacionadas à personalidade na formação da preferência do consumidor [8]. Além disso, vale ressal- 
tar as possiveis estratégias de marketing adotadas a partir da segmentação de mercado, facilitada pela identificação das diferenças individuais do consumidor através da variável NFC.

TABELA 3. Equivalência de escala: concordância entre as versões adaptada para o português e inglesa do questionário NFC.

\begin{tabular}{ll}
\hline Variável & Proporção de concordância \\
\hline Par 1 (Q 1) & 0,93 \\
Par 2 (Q 2) & 0,90 \\
Par 3 (Q 3) & 0,89 \\
Par 4 (Q 4) & 0,92 \\
Par 5 (Q 5) & 0,88 \\
Par 6 (Q 6) & 0,83 \\
Par 7 (Q 7) & 0,83 \\
Par 8 (Q 8) & 0,89 \\
Par 9 (Q 9) & 0,88 \\
Par 10 (Q 10) & 0,83 \\
Par 11 (Q 11) & 0,95 \\
Par 12 (Q 12) & 0,93 \\
Par 13 (Q 13) & 0,86 \\
Par 14 (Q 14) & 0,92 \\
Par 15 (Q 15) & 0,83 \\
Par 16 (Q 16) & 0,86 \\
Par 17 (Q 17) & 0,78 \\
Par 18 (Q 18) & 0,86 \\
\hline
\end{tabular}

$\mathrm{Q}=$ questão no instrumento Need for Cognition.

\section{4 - CONCLUSÕES}

O instrumento Need for Cognition disponibilizado para o português constitui uma ferramenta bastante útil que poderá ser utilizada em estudos de consumidor com indivíduos usuários da língua portuguesa.

\section{5 - REFERÊNCIAS}

[1] BADIA, X.; GUTIÉRREZ, F.; WIKLUND, I.; ALONSO, J. Validity and reliability of the Spanish version of the psychological general well-being index. Quality of Life Research, v.5, p.101-108, 1996.

[2] BEARDEN, W. O. ; NETEMEYER, R.G., MOBLEY, M.F. Handbook of Marketing Scales. Sage Publications, London, 1994.

[3] CACIOPPO, J.T.; PETTY, R.E.; KAO, C.F; RODRIGUEZ, R. Central and peripheral routes to persuasion: na individual difference perspective. J. Personality and Social Psychology, v.51, p.1032-1043, 1986.

[4] CACIOPPO, J.T.; PETTY, R.E.; KAO, C.F. The efficient assessment of Need for Cognition. J. of Personality Assessment, v.48, p.306-307, 1984.

[5] CACIOPPO, J.T. \& PETTY, R.E. The need for cognition. J. of Personality and Social Psychology, v.42, n.1, p.116131, 1982.

[6] DELIZA, R. The effects of expectation on sensory perception and acceptance. Reading, UK, 1996. 198p. Food Science and Technology Department, University of Reading.

[7] FRANCIS, LS; YAACOV, JK; JONES, SH. The reability and validity of the Hebrew version of the computer attitude scale. Computers \& Education, v.35, p. 149-159, 2000.

[8] HAUGTVEDT, C. P. ; PETTY, R.E.; CACIOPPO, J.T. Need for cognition and advertising: understanding the role of personality variables in consumer behavior. $\mathbf{J}$. Consumer Psychology, v.1, n.3, p. 239-260, 1992.

[9] PRIETO, A. J. A method for translation of instruments to other languages. Adult Education Quaterly, v.43, n.1, p.1-14, 1992.

[10] OQUENDO M.A; BACA-GARCIA, E.; GRAVER,R.; MORALES,M.; MONTALVAN, V.; MANN, J.J. Spanish adaptation of the reasons for living inventory. Hispanic J. of Behavioral Sciences, v.22, n.3, p.369-380, 2000.

[11] SON, G.R.; ZAUSZNIEWSKI, J.Á.; WYKLE, M.L.; PICOT, S.J.F. Translation and validation of caregiving satisfaction scale into Korean. Western J. of Nursering Research, v.22, n.5, p.609-622, 2000.

\section{6 - AGRADECIMENTOS}

Aos tradutores que gentilmente colaboraram na elaboração da versão em português, assim como na discussão para refinamento da ferramenta.

Órgãos financiadores: EMBRAPA. 